\title{
Lock-In Thermography to Analyze Plasmonic Nanoparticle Dispersions
}

\author{
Lukas Steinmetz, Patricia Taladriz-Blanco,* Christoph Geers, Miguel Spuch-Calvar, \\ Mathias Bonmarin, Sandor Balog, Barbara Rothen-Rutishauser, and Alke Petri-Fink**
}

The physicochemical properties of nanoparticles (NPs) strongly rely on their colloidal stability, and any given dispersion can display remarkably different features, depending on whether it contains single particles or clusters. Thus, developing efficient experimental methods that are able to provide accurate and reproducible measures of the NP properties is a considerable challenge for both research and industrial development. By analyzing different NPs, through size and concentration, it is demonstrated that lock-in thermography, based on light absorption and heat generation, is able to detect and differentiate the distinct aggregation and re-dispersion behavior of plasmonic NPs, e.g., gold and silver. Most importantly, the approach is nonintrusive and potentially highly cost-effective compared to standard analytical techniques. irradiation. ${ }^{[9,10]}$ Therefore, the heating effect of a NP becomes stronger upon light excitation at its localized surface plasmon resonance (LSPR) wavelength and can be precisely tuned by varying the size and shape of the NP. ${ }^{[11-14]}$

With the rise of thermoplasmonics, both industry and academia call for appropriate tools to ensure reliable detection and analysis of plasmonic nanoheaters. The latter becomes much more challenging if the NPs show signs of aggregation, which can strongly alter their behavior and performance, and is therefore an important factor to analyze. ${ }^{[13,15,16}$ Hence, determining NP aggregation is of crucial importance in the case of thermo-

The thermoplasmonic properties of metal nanoparticles (NPs), induced by their enhanced light absorption, are nowadays used to fabricate nanoheaters with applications in photothermal therapy, imaging, nanochemistry, and optofluidics. ${ }^{[1-4]}$ When a metal NP is irradiated, the conductive free electrons on the surface of the NP oscillate in a collective manner in response to the electromagnetic field, resulting in enhanced absorption and scattering resonances. ${ }^{[2,5-7]}$ While part of the incident light is scattered, the other is absorbed and concentrated on the surface of the NP. The absorbed light is transferred into heat with high efficiency and dissipated to the surrounding medium within picoseconds. ${ }^{[8]}$ Due to the enhanced optical crosssections of NPs in comparison to their geometrical cross-section, it is possible to locally generate high temperatures upon light

L. Steinmetz, Dr. P. Taladriz-Blanco, Dr. C. Geers, Dr. M. Spuch-Calvar, Dr. S. Balog, Prof. B. Rothen-Rutishauser, Prof. A. Petri-Fink Adolphe Merkle Institute

University of Fribourg

Chemin des Verdiers 4, 1700 Fribourg, Switzerland

E-mail: patricia.taladrizblanco@unifr.ch; alke.fink@unifr.ch

Dr. M. Bonmarin

School of Engineering

Zurich University of Applied Sciences

Technikumstrasse 9, 8400 Winterthur, Switzerland

Prof. A. Petri-Fink

Chemistry Department

University of Fribourg

Chemin du Musée 9, 1700 Fribourg, Switzerland plasmonics, as the plasmon resonance conditions drastically change. ${ }^{[17-19]}$ Another important parameter to consider refers to particle size and concentration, as potential applications rely on precise control and fine-tuning of these factors. ${ }^{[9,20]}$

Among other techniques, electron microscopy, light-scattering techniques, or UV-Vis spectroscopy allows researchers to analyze nanomaterials and their colloidal stability. ${ }^{[21,22]}$ However, every method has its strengths and limitations and therefore, new complementary approaches need to be developed and tested to explore the physicochemical properties of NPs. These new techniques either can overcome the limitations of existing techniques or can be used in combination with them. ${ }^{[23]}$ Lock-in thermography (LIT) is a nondestructive and nonintrusive infrared imaging technique, which allows to accurately measure temperature variations in the millikelvin range generated by stimuli-responsive materials, reacting to a specific excitation. ${ }^{[24-26]}$ The detection of such small temperature signals is achieved by applying a modulated excitation signal and recording numerous cycles. The method rejects slowly varying artifacts due to its averaging nature and significantly improves the signal-to-noise ratio. ${ }^{[25,26]}$ Previous studies have shown that LIT is a promising tool for the detection and characterization of superparamagnetic NPs in an alternating magnetic field or light-stimulated polydopamine NPs. ${ }^{[27-30]}$ These studies were performed under quasi-adiabatic conditions, and influences of evaporation or undesired phase transformations could be neglected due to the low amount of generated thermal energy. ${ }^{[28]}$ As the method uses a modulated signal to excite NPs, LIT can be adapted to analyze heat generated by different types of NPs by changing the stimulation source. While using a magnetic coil to excite superparamagnetic NPs, plasmonic NPs, 


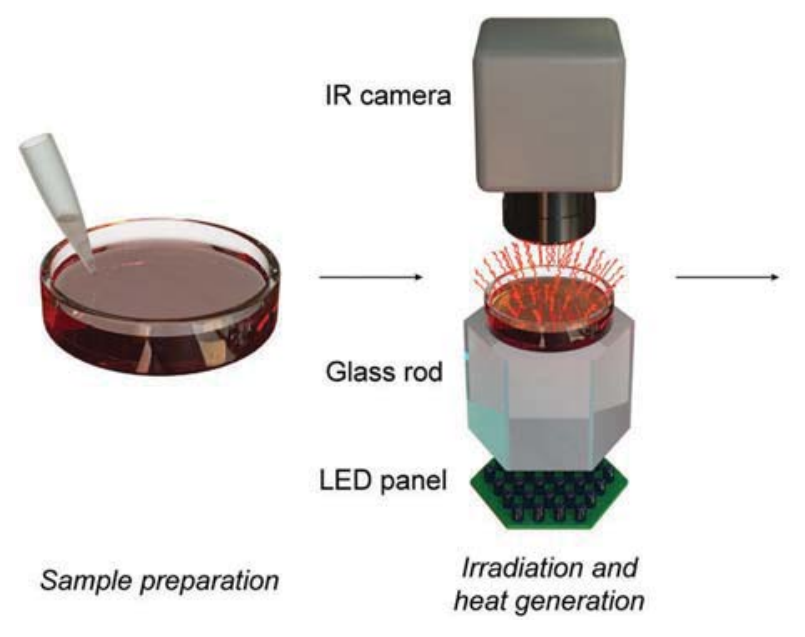

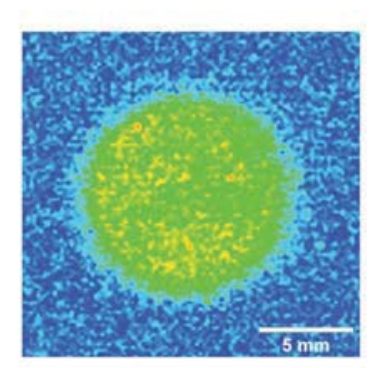

Heat map analysis

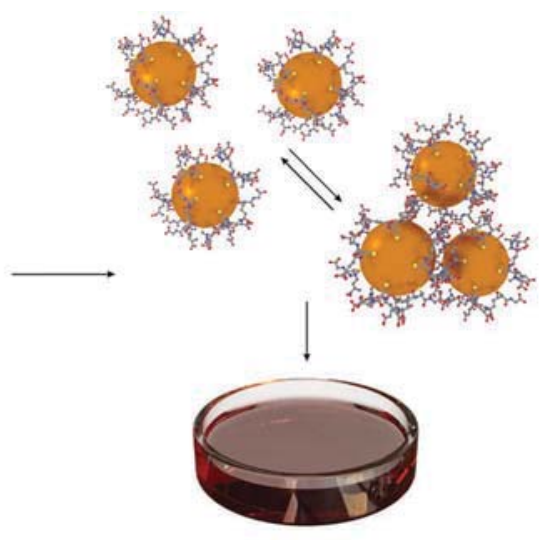

Feedback on colloidal stability

Figure 1. Principle of LIT measurements using light as the stimulation source. Due to the excitation, photons are absorbed by plasmonic NPs resulting in generated thermal energy and an elevated temperature of the particle dispersion. The heating depends on various material properties, such as NP size, shape, colloidal stability, and concentration. Therefore, thermography analysis can provide in situ information about material properties.

such as gold (Au) or silver (Ag), can be stimulated by applying a modulated illumination in the visible and near-infrared range. An integrated multi-wavelength light source allows to swiftly change between desired excitation wavelengths.

In comparison to standard characterization techniques, LIT combines numerous practically relevant advantages. In general, while using microscopy techniques we observe 2D projections of $3 \mathrm{D}$ objects, samples are limited to be very thin and drying effects are very common, whereas LIT allows the analysis of liquid and solid samples without any complex sample preparation. ${ }^{[31]}$ Furthermore, the area of detection in LIT is not limited to a small sample volume fraction, the method is nonintrusive and the sample can be recovered after the analysis. Considering that only the light absorbed by the NPs is converted into heat differentiates LIT from light-scattering techniques, ${ }^{[32]}$ as the main advantage of LIT relies on the flexibility to adapt the applied trigger signal to match with the plasmonic resonance of the NPs. In addition, only very low amounts of sample material ( $40 \mu \mathrm{L}$ per sample were used in this study) are needed to perform a LIT measurement, representing an advantage over common UV-Vis spectroscopy. Other techniques based on thermal detection, such as thermophoresis, ${ }^{[21]}$ or thermal lens microscopy, ${ }^{[33]}$ and photothermal heterodyne imaging ${ }^{[34]}$ based on lock-in detection, require expensive setups and experienced users. In contrast, LIT measurements can be conducted within minutes, which establishes LIT as a time and cost-effective complementary method to analyze NPs.

In this study, we employed LIT as a method to first study different NP sizes and concentrations in water. This enabled us to test suitable sample systems and their effect on the NP heat generation. In a second step, we monitored the heat generated during the aggregation of $\mathrm{Au}$ and AgNPs to show how changes in colloidal stability can be detected by using LIT.

Both investigated NP types are commonly used in research and industrial applications due to their excellent plasmonic properties and were therefore used as model particles. ${ }^{[5,20,35]}$ We observed a linear dependency for amplitude signal and concentration, and a significant difference in heat generation for 15 and $42 \mathrm{~nm}$ AuNPs. Furthermore, we were able to discern between dispersed and aggregated glutathione (GSH)-functionalized $\mathrm{Au}$ and AgNPs, due to their characteristic heat generation. Our results highlight the applicability of LIT to swiftly and accurately screen plasmonic NPs and distinguish between their aggregation and re-dispersion behavior. An overview of the LIT measurement principle is depicted in Figure 1.

Throughout this manuscript and in agreement with IUPAC terminology, we used the term "aggregated" to describe samples, which are in a nondispersed state. ${ }^{[36]}$

Figure 2 shows the concentration-dependent heat generation of 15 and $42 \mathrm{~nm}$ AuNPs upon irradiation with a lightemitting diode (LED) source centered at 525 and $660 \mathrm{~nm}$. With an increase in Au concentration from 0.3 to $0.6 \times 10^{-3} \mathrm{M}$,

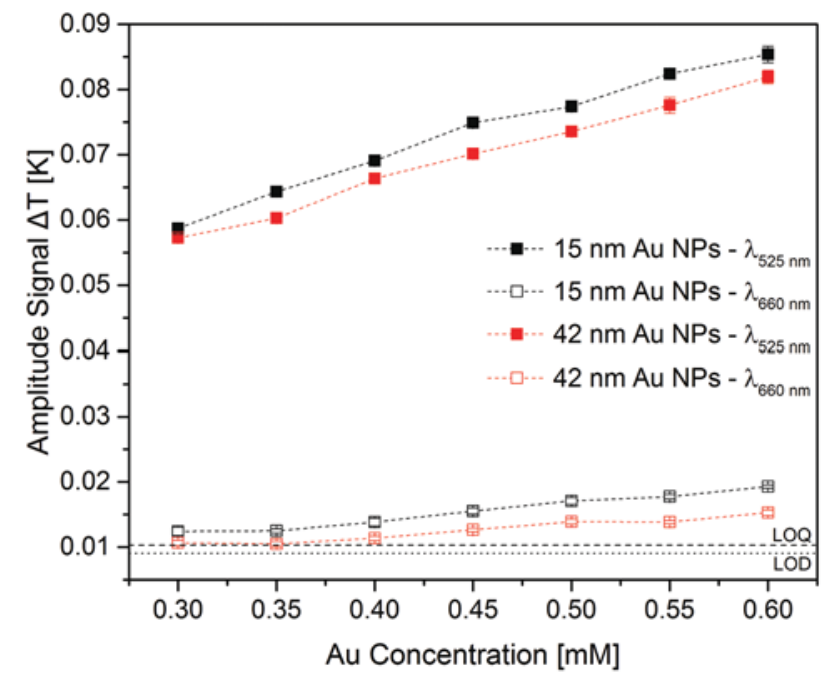

Figure 2. Heat generation of 15 and $42 \mathrm{~nm}$ AuNPs at $\lambda=525 \mathrm{~nm}$ and $\lambda=660 \mathrm{~nm}$ for different Au concentrations. A linear increase in heating power has been observed throughout the analyzed concentration range for both sizes. Smaller NPs generate thermal energy more efficiently than larger particles, as LSPR interactions are present to a greater extend. The amount of generated heat is reduced for both NP sizes at higher wavelengths, far away from optimal LSPR conditions. 
a linear increase in amplitude signal for both NP sizes was observed. Moreover, for both wavelengths, smaller particles displayed slightly higher amplitude signals at the same Au concentrations.

When irradiating plasmonic NPs with light, the absorbed energy is converted into heat with high efficiency and transferred to the environment. ${ }^{[1,8,37]}$ Therefore, the heat generation is especially strong when the wavelength of the light source is close to the wavelength for which the absorption cross-section of the NP is maximum. ${ }^{[13]}$ Figure S1 in the Supporting Information shows the theoretical extinction, scattering, and absorption cross-sections from 300 to $800 \mathrm{~nm}$ for the investigated NPs. ${ }^{[38]}$ Table S1 in the Supporting Information summarizes the theoretical values and shows the absorption efficiency values, defined as the ratio between the absorption and extinction cross-sections, at the irradiation wavelengths. When irradiating AuNPs at $\lambda=525 \mathrm{~nm}$, theoretical calculations based on the Mie theory show that, for the $15 \mathrm{~nm}$ NPs, $99 \%$ of the extinction cross-section is absorption, while for the $42 \mathrm{~nm} \mathrm{NPs}$, the absorption contribution decreases to $96 \%$. The remaining light is scattered and does not contribute to any heat generation. Thus, the higher amplitude signals of the smaller AuNPs can be explained. ${ }^{[7]}$ When the system is illuminated at $\lambda=660 \mathrm{~nm}$, the amplitude signal is significantly lower for both particle sizes. This effect can be observed, as the excitation wavelength is far away from the plasmonic resonance wavelength of the NPs $\left(\lambda_{\max }=519 \mathrm{~nm}\right.$ and $\lambda_{\max }=523 \mathrm{~nm}$, respectively) (Figure S2, Supporting Information). Therefore, heat generation is reduced due to the decrease in NP light absorption. It is worth noting that variations in heat generation caused by changes in concentrations as low as $0.05 \times 10^{-3} \mathrm{M}\left(=9.6 \mu \mathrm{g} \mathrm{mL}^{-1}\right)$ were reliably detected using LIT. The corresponding detection and quantification limits are depicted in Table S2 in the Supporting Information.

The aggregation and re-dispersion behavior of surface-coated $15 \mathrm{~nm}$ AuNPs and $17 \mathrm{~nm}$ AgNPs was analyzed by monitoring the change in heat generation. It has been demonstrated before that the surface functionalization of the investigated plasmonic NPs with DL-penicillamine (PEN), N-acetyl-DL-penicillamine (NAP), ${ }^{[39]}$ and $\mathrm{GSH}^{[18]}$ leads to $\mathrm{pH}$-controllable reversible aggregation of the NPs. This behavior is induced by the formation of hydrogen bonds, which occur if the acid groups of the thioaminoacid are protonated. The resulting particles will be referred to as AuNPs@GSH and AgNPs@GSH, respectively, in this manuscript, and their thorough characterization can be found in Table S3 in the Supporting Information.

Figure 3A,B shows the evolution of the absorbance spectra of AuNPs@GSH and AgNPs@GSH dispersions upon changing the $\mathrm{pH}$ of the solution from basic to acidic. For both dispersions, the NP aggregation induced a significant red-shift of the plasmon resonance band. To further visualize the aggregation and re-dispersion behavior, transmission electron microscopy images have been taken (Figures S3 and S4, Supporting Information).

Figure 3C,D depicts the related reversible heat generation of GSH-functionalized NPs obtained by alternating the $\mathrm{pH}$ of the dispersion and upon irradiation with an LED source centered at 525 and $400 \mathrm{~nm}$, respectively. As mentioned before, only the absorbed light can be efficiently converted into heat.
In addition, we recorded the heat generated by the same NPs upon illumination with an LED source at a wavelength far away from the plasmon resonance $(\lambda=660 \mathrm{~nm}$ for AuNPs and $\lambda=525 \mathrm{~nm}$ for AgNPs) (Figure S5, Supporting Information). When irradiating at the maximum intensity of the extinction spectra ( $\lambda=525 \mathrm{~nm}$ for AuNPs and $\lambda=400 \mathrm{~nm}$ for AgNPs), the heat generated by the particles in a dispersed state is higher than in an aggregated state. Hence, the decrease of heat is attributed to the increased scattering when the particles are aggregated (Table S1, Supporting Information). An inverse trend was observed for the AuNPs excited at $660 \mathrm{~nm}$ (and AgNPs at $525 \mathrm{~nm}$ ), as the aggregated NPs still produce an increased amount of thermal energy, while the dispersed NPs generate less (Figure S5, Supporting Information). The aggregation-induced red-shift led to a change in absorbance intensity and, therefore, to an altered heat generation behavior. When particles are aggregated, they act as larger single NPs, therefore their scattering is increased. However, when comparing the experimental extinction spectra of the aggregated NPs with theoretical and experimental data of large NPs, the optical behavior of our aggregated $\mathrm{Au}$ and AgNPs is similar to NPs of approximately $200 \mathrm{~nm}$ in diameter. ${ }^{[40,41]}$ Therefore, when irradiating the NP dispersion at $660 \mathrm{~nm}$ for AuNPs and $525 \mathrm{~nm}$ for AgNPs, our system is being excited close to the maximum absorption cross-section. The latter is the result of the increased heat generation for aggregated NPs. It is important to mention that while it was possible to fully reverse the induced aggregation of the AuNPs (Figure 3C), a reduction of light absorbance and consequently, in heat generation was observed after one cycle for re-dispersed AgNPs upon excitation at $400 \mathrm{~nm}$ (Figure 3D). This observation was attributed to the oxidation of AgNPs, which led to a decrease in plasmon resonance (Figure 3B). Nevertheless, the obtained amplitude signals demonstrated the reproducibility of LIT measurements for all dispersed and aggregated samples.

In our study, we tested LIT as a tool to distinguish between dispersed and aggregated plasmonic NPs by measuring their heat generation. We highlighted the sensitivity of LIT toward varying NP sizes and concentrations, as the method is able to reliably distinguish between 15 and $42 \mathrm{~nm}$ AuNPs and resolve differences in Au concentration in $0.05 \times 10^{-3} \mathrm{M}\left(=9.6 \mu \mathrm{g} \mathrm{mL}^{-1}\right)$ steps. These plasmonic NPs generate heat upon stimulation with light, which correlates with their enhanced absorbance characteristics. LIT allows to measure even low amounts of generated thermal energy in a nonintrusive and nondestructive way. In contrast to dispersed NPs, aggregated NPs exhibit a shift in their absorbance spectra, resulting in altered heat generation upon irradiation. Depending on the applied wavelength, the amount of released thermal energy may vary, e.g., aggregated AuNPs exhibited decreased heat generation when compared to the dispersed $15 \mathrm{~nm} \mathrm{NPs}$ at $\lambda=525 \mathrm{~nm}$, but the opposite was observed at $\lambda=660 \mathrm{~nm}$. Similar trends were obtained for $17 \mathrm{~nm}$ AgNPs, but for a stimulation of $\lambda=400 \mathrm{~nm}$ and $\lambda=525 \mathrm{~nm}$. Therefore, great care should be taken when selecting the excitation wavelength. Furthermore, we demonstrated that our results are reproducible, even upon cycling between dispersed and aggregated NPs. Reversible aggregation was achieved by NP functionalization with GSH and subsequent alteration of the $\mathrm{pH}$. Applications of LIT could 

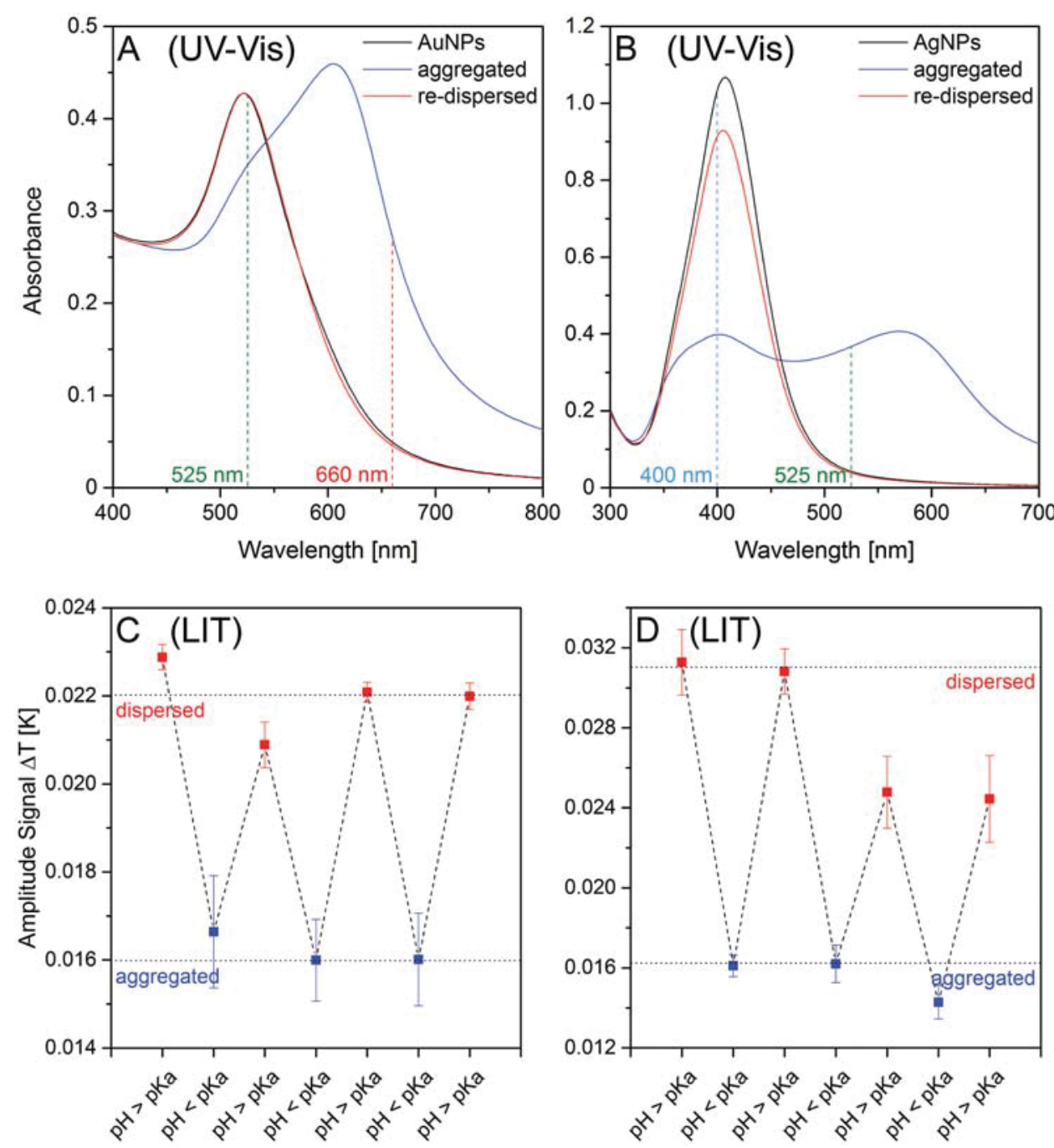

Figure 3. $A, B)$ UV-vis spectra and $C, D)$ LIT amplitude signals of dispersed, aggregated, and re-dispersed $G S H$-functionalized $A, C) 15 \mathrm{~nm} A u$ and $B, D) 17 \mathrm{~nm}$ Ag NPs. A red-shift in absorbance is observed upon the addition of acid, causing NP aggregation (A,B: blue spectrum). Increasing the pH to neutral results in NP re-dispersion (A,B: red spectrum). Excitation wavelengths (525/660 nm for AuNPs and 400/525 nm for AgNPs) were derived from these spectra and were used for all LIT experiments. Amplitude signals of well-dispersed NPs result in higher intensities than aggregated NPs for an excitation wavelength of C) $525 \mathrm{~nm}$ for AuNPs and D) $400 \mathrm{~nm}$ for AgNPs. By altering the pH (high: > pKa, low: < pKa) NPs can be aggregated and re-dispersed over several cycles.

possibly range from lab-based NP characterization to industrial batch-to-batch reproducibility measurements. When it comes to the analysis of thermoplasmonics, LIT combines advantages of spectroscopic and calorimetric techniques, while no complex sample preparation and only a few $\mu \mathrm{L}$ of sample volume are needed. Additionally, measurements are performed within minutes, which renders the method effort, time, and cost effective. Overall, LIT stands out due to its versatility, rapid detection system, and straightforward approach. Its wide field of view, which allows to analyze the entire sample volume at once, is advantageous compared to many other techniques, such as UV-Vis. For the future, an extended range of NP systems with varying chemistry, shapes, and in complex analytical environments should be investigated to demonstrate the validation of the method for different wavelengths and materials.

\section{Experimental Section}

To analyze NP heat generation, LIT measurements were conducted. A custom-made experimental setup was built, consisting of a homogenous LED-based light source (AN178_2 61 LED module, ADOM), an infrared camera (Onca-MWIR-InSb-320, XenICs) mounted on a standard microscope stand (Leica Microsystems), and a personal computer as a processing unit. The camera InSb array $(320 \times 256$ pixels $)$ operated in the mid-IR range $(3-5 \mu \mathrm{m})$ and was capable of capturing full frame images at a rate up to $200 \mathrm{~Hz}$. A camera-link frame grabber was used to transfer the acquired images in real-time to a personal computer for further data processing with a custom-made LabVIEW-based software. ${ }^{[27]}$ In brief, the software demodulated the acquired infrared images according to the digital lock-in principle to compute amplitude maps (in Kelvin) proportional to the sample generated heat. ${ }^{[26]}$

The LED module emitted excitation light at various wavelengths $(400$, 525 , and $660 \mathrm{~nm}$ ). To ensure homogenous spatial illumination of the sample, a light-homogenizing glass rod (N-BK7, Edmond Optics) was placed between the LED panel and the sample. All measurements were 
performed at a modulation frequency of $1 \mathrm{~Hz}$ and an excitation current of $150 \mathrm{~mA}$ for $400 \mathrm{~nm}$ and of $200 \mathrm{~mA}$ for $525 \mathrm{~nm}$ and $660 \mathrm{~nm}$ LEDs, resulting in power densities of $2,1.8$, and $5.7 \mathrm{~mW} \mathrm{~cm}{ }^{-2}$, respectively. The duration of one measurement was set to $60 \mathrm{~s}$ and kept constant for every sample.

Samples were prepared as an aqueous NP dispersion of $40 \mu \mathrm{L}$ at varying concentrations and measured five times consecutively. Custommade, spherical, and light-transparent sample holders made from polystyrene were used for all NP dispersions to reduce heat losses to the environment and ensure quasi-adiabatic conditions. ${ }^{[27]}$ The resulting amplitude images were analyzed (Image) v1.52b, NIH \& Origin 2016, OriginLab) to extract signal mean and standard deviation values.

A detailed description of the Experimental Section of this study can be found in the Supporting Information.

\section{Supporting Information}

Supporting Information is available

\section{Acknowledgements}

This work was supported by the Swiss National Science Foundation (Grant no. 200021_159803), the University of Fribourg, and the Adolphe Merkle Foundation. Parts of this work were supported by the National Center of Competence in Research Bio-Inspired Materials. The authors thank Nanolockin for the appropriation of their Celvin Alpha prototype and Dr. Kyle Rodriguez for proofreading the manuscript. The manuscript was written through contributions of all authors. All authors have given approval to the final version of the manuscript.

\section{Conflict of Interest}

The authors declare no conflict of interest.

\section{Keywords}

aggregation, lock-in thermography, nanoheaters, nanomaterial characterization, thermoplasmonics

[1] G. Baffou, R. Quidant, Laser Photonics Rev. 2013, 7, 171.

[2] K. Jiang, D. A. Smith, A. Pinchuk, J. Phys. Chem. C 2013, 117, 27073.

[3] D. Jaque, L. Martínez Maestro, B. del Rosal, P. Haro-Gonzalez, A. Benayas, J. L. Plaza, E. Martín Rodríguez, J. García Solé, Nanoscale 2014, 6, 9494.

[4] N. S. Abadeer, C. J. Murphy, J. Phys. Chem. C 2016, 120, 4691.

[5] A. O. Govorov, H. H. Richardson, Nano Today 2007, 2, 30.

[6] G. Baffou, R. Quidant, F. J. García De Abajo, ACS Nano 2010, 4, 709.

[7] A. Arbouet, D. Christofilos, N. Del Fatti, F. Vallée, J. R. Huntzinger, L. Arnaud, P. Billaud, M. Broyer, Phys. Rev. Lett. 2004, 93, 2.

[8] D. K. Roper, W. Ahn, M. Hoepfner, J. Phys. Chem. C 2007, 111, 3636.

[9] P. K. Jain, K. S. Lee, I. H. El-Sayed, M. A. El-Sayed, J. Phys. Chem. B 2006, 110, 7238 .
[10] V. K. Pustovalov, Laser Phys. Lett. 2005, 2, 401

[11] V. Amendola, R. Pilot, M. Frasconi, O. M. Marago, M. A. Iati, J. Phys.: Condens. Matter 2017, 29, 203002.

[12] G. Baffou, R. Quidant, C. Girard, Appl. Phys. Lett. 2009, 94, 153109.

[13] H. Chen, L. Shao, T. Ming, Z. Sun, C. Zhao, B. Yang, J. Wang, Small 2010, 6, 2272.

[14] L. M. Liz-Marzán, Mater. Today 2004, 7, 26.

[15] W. Zhang, in Advances in Experimental Medicine and Biology (Eds: D. G. Capco, Y. Chen), Springer,Dordrecht, Netherlands 2014, pp. 19-43.

[16] Z. Qin, Y. Wang, J. Randrianalisoa, V. Raeesi, W. C. W. Chan, W. Lipinski, J. C. Bischof, Sci. Rep. 2016, 6, 1.

[17] D. H. Tsai, L. F. Pease, R. A. Zangmeister, M. J. Tarlov, M. R. Zachariah, Langmuir 2009, 25, 140.

[18] I. I. S. Lim, D. Mott, W. Ip, P. N. Njoki, Y. Pan, S. Zhou, C. J. Zhong, Langmuir 2008, 24, 8857.

[19] J. Stehr, C. Hrelescu, R. A. Sperling, G. Raschke, M. Wunderlich, A. Nichtl, D. Heindl, K. Kürzinger, W. J. Parak, T. A. Klar, S. J. Feldmann, Nano Lett. 2008, 8, 619.

[20] M. Daniel, D. Astruc, Chem. Rev. 2004, 104, 293.

[21] R. A. Sperling, T. Liedl, S. Duhr, S. Kudera, M. Zanella, C. A. J. Lin, W. H. Chang, D. Braun, W. J. Parak, J. Phys. Chem. C 2007, 111, 11552

[22] W. Ni, R. A. Mosquera, J. Párez-Juste, L. M. Liz-Marzán, J. Phys. Chem. Lett. 2010, 1, 1181.

[23] S. Mourdikoudis, R. M. Pallares, N. T. K. Thanh, Nanoscale 2018, 10, 12871.

[24] D. Wu, G. Busse, Rev. Gen. Therm. 1998, 37, 693.

[25] S. Huth, O. Breitenstein, A. Huber, D. Dantz, U. Lambert, F. Altmann, Solid State Phenom. 2002, 82-84, 741

[26] O. Breitenstein, W. Warta, M. C. Schubert, Lock-in Thermography, Springer International Publishing, New York 2018

[27] C. A. Monnier, M. Lattuada, D. Burnand, F. Crippa, J. C. MartinezGarcia, A. M. Hirt, B. Rothen-Rutishauser, M. Bonmarin, A. Petri-Fink, Nanoscale 2016, 8, 13321.

[28] C. A. Monnier, F. Crippa, C. Geers, E. Knapp, B. Rothen-Rutishauser, M. Bonmarin, M. Lattuada, A. Petri-Fink, J. Phys. Chem. C 2017, 121, 27164.

[29] P. Lemal, C. Geers, C. A. Monnier, F. Crippa, L. Daum, D. A. Urban, B. Rothen-Rutishauser, M. Bonmarin, A. Petri-Fink, T. L. Moore, J. Magn. Magn. Mater. 2017, 427, 206.

[30] D. Hauser, M. Estermann, A. Milosevic, L. Steinmetz, D. Vanhecke, D. Septiadi, B. Drasler, A. Petri-Fink, V. Ball, B. Rothen-Rutishauser, Nanomaterials 2018, 8, 1065.

[31] B. Michen, C. Geers, D. Vanhecke, C. Endes, B. Rothen-Rutishauser S. Balog, A. Petri-Fink, Sci. Rep. 2015, 5, 9793.

[32] C. Geers, L. Rodriguez-Lorenzo, D. A. Urban, C. Kinnear, A. Petri-Fink, S. Balog, Nanoscale 2016, 8, 15813.

[33] K. Uchiyama, A. Hibara, H. Kimura, T. Sawada, T. Kitamori, Jpn. J. Appl. Phys. 2000, 39, 5316.

[34] S. Berciaud, D. Lasne, G. A. Blab, L. Cognet, B. Lounis, Phys. Rev. B 2006, 73, 1 .

[35] S. Prabhu, E. K. Poulose, Int. Nano Lett. 2012, 2, 1.

[36] R. G. Jones, C. K. Ober, P. Hodge, P. Kratochvíl, G. Moad, M. Vert, Pure Appl. Chem. 2013, 85, 463

[37] G. Baffou, Photoniques 2018, S3, 42.

[38] R. Yu, L. M. Liz-Marzán, F. J. García de Abajo, Chem. Soc. Rev. 2017, 46, 6710.

[39] P. Taladriz-Blanco, N. J. Buurma, L. Rodríguez-Lorenzo, J. Pérez-Juste, L. M. Liz-Marzán, P. Hervés, J. Mater. Chem. 2011, $21,16880$.

[40] N. G. Bastús, J. Comenge, V. Puntes, Langmuir 2011, 27, 11098.

[41] N. G. Bastús, F. Merkoçi, J. Piella, V. Puntes, Chem. Mater. 2014, 26, 2836. 